\title{
Effect of Revaccination with Recombinant Hepatitis B Virus Vaccine Containing Higher Antigen Concentration in Non-responder Children
}

\author{
Riska Habriel Ruslie ${ }^{1 *}$, Gontar Alamsyah Siregar ${ }^{2}$ \\ ${ }^{1}$ Department of Child Health, Faculty of Medicine, Universitas Prima Indonesia, Medan, Indonesia; ${ }^{2}$ Department of Internal \\ Medicine, Faculty of Medicine, Universitas Sumatera Utara, Medan, Indonesia
}

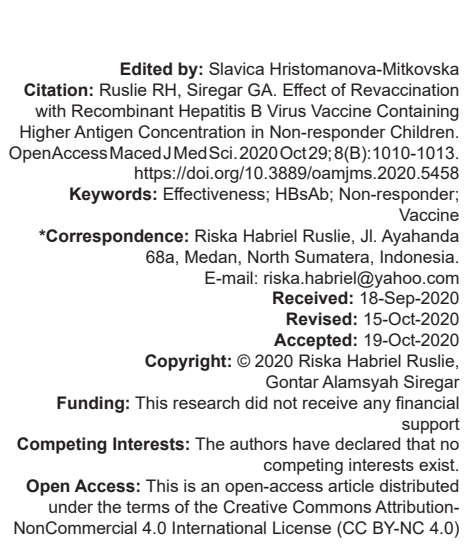

\section{Introduction}

Hepatitis B still becomes a global health problem [1], [2]. Approximately 700 million adults have a history of prior hepatitis $B$ and 120 million individuals are chronic hepatitis B surface antigen (HBsAg) carriers [3] It is estimated that 500,000 until 1.2 million global deaths are related to hepatitis $B$. The prevalence of hepatitis $B$ is higher in Asia compared to Europe [1]. Most developed countries have low hepatitis $B$ endemicity and the transmission usually occurs horizontally from high-risk behaviors such as homosexual and drug user, blood transfusion, hemodialysis, and healthcare workers [2]. Vaccination is a preventive measure against hepatitis $B$ virus infection and its complications [4], [5]. Vaccination against hepatitis $B$ has been invented for a few decades ago. Universal childhood vaccination program involving this vaccine has greatly reduced the rate of both acute and chronic hepatitis B virus (HBV) infections [3], [6].
In the Netherlands, the vaccine should be administered at 0,1 , and 2 months [6]. In Indonesia, routine vaccination against hepatitis $B$ utilizes a vaccine with a dose of $10 \mu \mathrm{g}$. Each person should receive a total of 3 doses at 0,1 , and 6 months [7]. Protective response following vaccination is marked by anti-hepatitis B surface antigen (HBsAb) titer of $10 \mathrm{IU} / \mathrm{L}$ or higher [1], [6]. If someone has reached protective HBsAb level, he/she will have a lower risk of hepatitis $B$ for at least 10 years [5]. It is reported that $5-30 \%$ person does not have a protective response after routine vaccination. They are known as non-responders [3], [4], [6], [8] while those who had HBsAb titer between 10 and $100 \mathrm{IU} / \mathrm{L}$ are called low responders [3], [4], [8]. Another literature defines non-responder as a subject who is unable to produce protective HBsAb titer despite receiving 2 series of $\mathrm{HBV}$ vaccination after acute or chronic hepatitis $B$ have been excluded and low responder as a non-responder who experiences seroconversion after the second series of HBV vaccination [7]. Non-responders are at 
high risk of HBV infection and its complications. They also become sources of hepatitis B spreading [6].

Repeated vaccination or revaccination is recommended for non-responders to overcome the risks [6], [7], [9]. There are several methods in conducting revaccination including giving additional dose at varying times, repeating standard schedule, administering a double dose, giving vaccine with higher antigen content, and administering vaccine through the intradermal route [5]. Our study aimed to determine the effect of revaccination with HBV vaccine containing higher antigen concentration in non-responder children.

\section{Methods}

This prospective study was conducted in Universitas Sumatera Utara Hospital and several private clinics in Medan, Indonesia from January 2018 until December 2019. Subjects were children aged 9-15 months previously vaccinated with three doses of recombinant $\mathrm{HBV}$ vaccine according to the governmental program at 0,1 , and 6 months old. Exclusion criteria were children suffering from acute or chronic hepatitis B, consuming immunosuppressant medications, and having conditions which alter immune function such as acquired immune deficiency syndrome. Subjects were obtained using a total sampling method. Demographic and clinical characteristics were gathered from each subject. Determination of serum HBsAb titer was conducted using HBsAb commercial kit (Diapro, Italy) according to the manufacturer's instruction. A venous blood sample was drawn and centrifugated to obtain the serum. Sera were kept at a temperature of $-20^{\circ} \mathrm{C}$ until the procedure was performed. Subjects with serum $\mathrm{HBsAb}$ titer of $<10 \mathrm{IU} / \mathrm{L}$ were classified into non-responders and further underwent revaccination with 3 doses of Engerix-B (GlaxoSmithKline) containing $20 \mu \mathrm{g} \mathrm{HBsAg}$ at the following 0, 1, and 6 months. The revaccination series was repeated until all subjects showed seroconversion into the protective level. After each series, repeated serum HBsAb titer measurements were conducted. Data analysis was done with statistical software and the results will be presented in tables. This study was approved by the Health Research Ethical Committee, Medical School, Universitas Sumatera Utara.

\section{Results}

A total of 400 children fulfilled the inclusion criteria of this study. Mean age of subjects was 12.1 (SD 2.64) months. Gender distribution was almost similar with slight female domination. A total of $79 \%$ subject had a normal nutritional status. The amount of nonresponders in this study was $36(9 \%)$ children (Table 1$)$. The 36 children followed revaccination program, as mentioned above.

Table 1: Baseline characteristics of subjects

\begin{tabular}{ll}
\hline Characteristics & $\mathrm{n}=400$ \\
\hline Mean age, months (SD) & $12.1(2.64)$ \\
Gender, $\mathrm{n}(\%)$ & $184(46.0)$ \\
$\quad$ Male & $216(54.0)$ \\
Female & \\
Nutritional status, $\mathrm{n}(\%)$ & $48(12.0)$ \\
$\quad$ Underweight & $316(79)$ \\
$\quad$ Normal & $36(9)$ \\
$\quad$ Overweight & \\
Immune response, $\mathrm{n}(\%)$ & $36(9.0)$ \\
$\quad$ Non-responder & $364(91.0)$ \\
$\quad$ Protective level &
\end{tabular}

Of 36 non-responder children who underwent the first series of revaccination, 32 children achieved protective serum HBsAb titer. The rest 4 children followed the second series of revaccination. In the end of the series, serum HBsAb titer of all subjects was in protective level (Table 2).

Table 2: Immune response of subjects after the first and second series of revaccination

\begin{tabular}{lll}
\hline Immune response & $\begin{array}{l}\text { After the first series of } \\
\text { revaccination }\end{array}$ & $\begin{array}{l}\text { After the second series of } \\
\text { revaccination }\end{array}$ \\
\hline Non-responder, $\mathrm{n}(\%)$ & $4(11.1)$ & $0(0.0)$ \\
Protective level & $32(88.9)$ & $4(100.0)$ \\
\hline
\end{tabular}

\section{Discussion}

Hepatitis B is a disease caused by hepatitis $B$ virus infection which may progress to chronic disease and cause severe complications such as liver cirrhosis and hepatocellular carcinoma [2], [10], [11]. Vaccine against HBV was introduced in the early 1980s and WHO urged all countries to involve the vaccine into their immunization programs [1], [8], [11]. All HBV vaccines contain $\mathrm{HBsAg}$, a protein from hepatitis $B$ virus envelope. Hepatitis $B$ surface antigen is expected to activate lymphocyte cells through antigen-presenting cells and preserve immunity in a longer period. There are 2 types of HBV vaccine: Plasma-derived vaccine and recombinant vaccine. Adjuvants are added to improve the immunogenicity of the vaccine [8]. Complete schedule of vaccination against HBV (3 or 4 doses according to regional protocol) gives safe and effective protection. However, a particular subject does not produce protective HBsAb even after complete vaccination [10]. A study from Iran involving children and adolescents aged 6-18 years reported that the response to HBV vaccine was only $38.5 \%$ [1]. Data from Bangladesh reported that $88.67 \%$ vaccinated individuals had protective HBsAb titer [2]. About 4\% vaccinated newborns with $\mathrm{HBV}$ vaccine does not reach protective antibody level [1], [12] where $0.4 \%$ does not respond even after revaccination while the rest $3.6 \%$ 
achieves protective antibody titer [12]. The result in our study was similar to the literature above. As many as $91 \%$ children receiving routine hepatitis $B$ vaccination reached protective serum $\mathrm{HBs} A b$ titer. The rate of nonresponder in our study was $9.0 \%$.

Poor response toward vaccination is associated with male gender, advanced age, obesity, smoking, genetic predisposition, type of vaccine, number of injection, subcutaneous administration of vaccine, adjuvant used, and vaccine handling [2], [4], [6], [7], [9]. Low ratio of $\mathrm{T}$ helper cell compared to $\mathrm{T}$ cytotoxic cell, high ratio of natural killer to T killer cell, and psychosocial stress shortly after vaccination is other risk factors for unsatisfied respond toward vaccination [10]. Older age is associated with suboptimal response toward HBV vaccine due to waning of immunity [1], [8]. Subcutaneous injection may decrease antigen-presenting cell's activation and inhibit antigen presentation to lymphocytes [7]. Obesity is associated with decreased number and function of lymphocytes [9]. Higher body weight also hampers immune response from decreased vaccine absorption in adipose tissue [1]. A case report described that obese women who had received six doses of $\mathrm{HBV}$ vaccine failed to achieve protective HBsAb titer until she underwent gastroplasty Roux-en-Y gastric bypass to overcome her obesity. She needed 3 additional doses after the surgery to experience seroconversion [9]. Genetic susceptibility in $\mathrm{HBV}$ vaccine non-responsiveness is associated with HLA haplotypes such as DRB1*3, DRB1*7, DRB1*14, $\mathrm{DQB} 1{ }^{*} 0604, \mathrm{DQA} 1{ }^{*} 0102$, and $\mathrm{DRB} 1 * 1302$ [8], [11]. The role HLA-C4A locus in the responsiveness toward $\mathrm{HBV}$ vaccination is hypothesized. The presence of C4AQ0 allele is associated with non-responsiveness to HBV vaccination even in the absence of $\mathrm{DRB} 1{ }^{*} 0301$, $\mathrm{DQB} 1{ }^{*} 02$, or $\mathrm{DRB} 1^{*} 0701$. The allele is expected to impair the function of complement and further antibody response to T-dependent antigen. This condition is observed in both adults and neonates [12]. Other literatures stated that the presence of HLA-DR2, HLA-DR3, HLA-DR7, HLA-DR14, and HLA-B8 was the genetic-associated risk factor for poor vaccination response [2], [4], [10]. There was no significant gender predisposition in our study. All subjects were also aged $<2$ years. Most subjects had a normal nutritional status.

A study evaluated revaccination response in non-responder adults after receiving 3 doses of Engerix-B or HBVaxpro-10. They were revaccinated by either Twinrix (GlaxoSmithKline), HBVaxPro40 (Merck Sharp \& Dohme), or Fendrix (GlaxoSmithKline) compared to Engerix-B (GlaxoSmithKline). Statistical analysis showed that the proportion of responders in HBVaxPro40 and Fendrix groups was higher compared to Engerix-B group as the control. HBsAb titer was also higher in both groups compared to control. This was probably caused by a higher antigen dose in Fendrix and HBVaxPro40. More potent adjuvant used in both vaccines might also contribute to higher response [6].
Clemens et al. studied a proportion of low- and nonresponder adults after hepatitis $B$ vaccination at 0 , 1 , and 6 months. They administered booster vaccine (Engerix-B, SmithKline Beecham Biologicals) with higher HBV antigen dose $(20 \mu \mathrm{g})$ at 2 months interval until the subjects had HBsAb titer of $\geq 100 \mathrm{mlU} / \mathrm{mL}$. After the $3^{\text {rd }}$ booster, all non-responders had HBsAb titer of $100 \mathrm{mIU} / \mathrm{mL}$ or higher [10]. Han et al. conducted a study in Guangdong and found that the rate of nonresponder and low responder of $3.1 \%$ and $28.9 \%$, respectively, in infants receiving 3 doses of routine HBV vaccination. The routine $\mathrm{HBV}$ vaccine used contains $5 \mu \mathrm{g}$ antigens. Revaccination was conducted using 3 doses of HBV vaccine with a higher content of antigen. All of the non-responders achieved low responder and responder status after revaccination [3]. The literature above confirmed the result of our study. We found that revaccination with HBV vaccine containing higher antigen concentration resulted in better immune response in non-responder children. However, we needed 2 series of revaccination to achieve serum $\mathrm{HBsAb}$ titer of $\geq 10 \mathrm{IU} / \mathrm{L}$ in all subjects who previously were non-responders.

In contrast with our study, Joukar et al. showed that a double dose of vaccine was more effective compared to a single dose in the early revaccination program. However, after the third dose of vaccine, both single and double dose gave similar effectiveness. All non-responsive subjects experienced seroconversion after the third dose of revaccination [5]. Their result was supported by several studies. A study in Bandung, Indonesia, showed that $5.5 \%$ of 144 children were non-responders. After receiving the second series of revaccination, all of them had protective $\mathrm{HBsAb}$ titer [7]. Cardell et al. administered 3 doses of combined hepatitis $A$ and $B$ vaccine as a revaccination program. After the third dose of vaccination, 95\% non-responders produced a protective level of HBsAb [11]. Ahad et al. reported that booster HBV vaccine significantly increased HBsAb titer [2].

Intradermal administration of $\mathrm{HBV}$ vaccine is suggested to be more effective in particular populations, including non-responders. Intradermal administration also requires less amount of antigen to induce protective immunity and may cut the cost of immunization [4]. This statement is supported by Cardell et al. Their study found that administration of hepatitis $B$ vaccine intradermally resulted in antibody production to a protective level in $68.3 \%$ study subjects. Administration of additional 1 booster vaccine increased the percentage to $88.9 \%$ [11]. However, the effectiveness of intradermal administration of HBV vaccine needs further study before being applied to non-responders [4].

The limitation of our study was that we could not rule out confounding factors non-responsiveness status such as genetic predisposition and vaccine handling. We also did not conduct follow-up after 
revaccination series to rule out the factors that might contribute to vaccination response. However, this was the first study in our region regarding the management of HBV vaccine non-responder children. In conclusion, revaccination with recombinant HBV vaccine containing higher antigen concentration in non-responder children is effective.

\section{References}

1. Jouneghani AS, Chaleshtori $M H$, Khoshdel A, Kheiri S, Farrokhi E, Khalafian $\mathrm{P}$, et al. Evaluation of response to hepatitis B vaccine in Iranian 6-18-year-old students. J Res Med Sci. 2017;22:116. https://doi.org/10.4103/jrms.jrms_204_17 PMid:29184574

2. Ahad MA, Alim MA, Guho A, Islam QT, Azad KA. Role of booster dose on antibody titer after recombinant hepatitis $B$ vaccination. J Med. 2009;10:67-76. https://doi.org/10.3329/jom.v10i2.2817

3. Han K, Shao X, Zheng $\mathrm{H}$, Wu C, Zhu J, Zheng X, et al. Revaccination of non- and low- responders after a standard three dose hepatitis B vaccine schedule. Hum Vaccin Immunother. 2012;8(12):1845-9. https://doi.org/10.4161/hv.21818 PMid:22906933

4. Filippelli M, Lionetti E, Gennaro A, Lanzafame A, Arrigo T, Salpietro $C$, et al. Hepatitis $B$ vaccine by intradermal route in non responder patients: An update. World J Gastroenterol. 2014;20(30):10383-94. https://doi.org/10.3748/wjg.v20. i30.10383

PMid:25132754

5. Joukar F, Mansour-Ghanaei F, Naghipour M, Asgharnezhad M. Immune responses to single-dose versus double-dose hepatitis $B$ vaccines in healthcare workers not responding to the primary vaccine series: A randomized clinical trial. Hepat Mon. 2016;16(2):e32799. https://doi.org/10.5812/hepatmon.32799

PMid:27148385

6. Raven SF, Hoebe CJ, Vossen AC, Visser LG, Hautvast JL, Roukens $A H$, et al. Serological response to three alternative series of hepatitis $B$ revaccination (Fendrix, Twinrix, and HBVaxPro-40) in healthy non-responders: A multicentre, open-label, randomised, controlled, superiority trial. Lancet Infect Dis. 2019;20(1):92-101. https://doi.org/10.1016/ s1473-3099(19)30417-7

7. Rusmil K, Fadlyana E, Bachtiar NS. Booster vaksinas hepatitis B terhadap anak yang non responder. Sari Pediatri. 2010;12(2):88-91. https://doi.org/10.14238/sp12.2.2010.88-91

8. Shouval D. Hepatitis B vaccines. J Hepatol. 2003;39 Suppl 1:S70-6.

PMid: 14708681

9. Dinelli MI, Moraes-Pinto MI. Seroconvertion to hepatitis B vaccine after weight reduction in obese non-responder. Rev Inst Med Trop Sao Paulo. 2008;50(2):129-30. https://doi. org/10.1590/s0036-46652008000200013 PMid:18488095

10. Clemens R, Sänger R, Kruppenbacher J, Höbel W, Stanbury W, Bock HL, et al. Booster immunization of low- and non-responders after a standard three dose hepatitis B vaccine schedule--results of a post-marketing surveillance. Vaccine. 1997;15(4):349-52. https://doi.org/10.1016/s0264-410x(96)00205-8 PMid:9141203

11. Cardell K. Studies on Hepatitis B Vaccination and Factors Associated with the Vaccine Response, Dissertation. Linköping: Linköping University Sweden; 2009

12. De Silvestri A, Pasi A, Martinetti M, Belloni C, Tinelli C, Rondini $G$, et al. Family study of non-responsiveness to hepatitis $B$ vaccine confirms the importance of HLA class III C4A locus. Genes Immun. 2001;2(7):367-72. https://doi.org/10.1038/ sj.gene.6363792

PMid:11704802 